\title{
An improved instrument for the in vivo detection of lead in bone
}

\author{
C L Gordon, D R Chettle, C E Webber
}

\begin{abstract}
An improved instrument for the fluorescence excitation measurement of concentrations of lead in bone has been developed. This is based on a large area high purity germanium detector and a point source of ${ }^{109} \mathrm{Cd}$. The source is positioned in a tungsten shield at the centre of the detector face such that $88 \mathrm{keV}$ photons cannot enter the detector directly. In vivo measurements are calibrated with plaster of Paris phantoms. Occupationally non-exposed men show a minimum detectable concentration of about $6 \mu \mathrm{g} / \mathrm{g}$ bone mineral. Measurements of tibia lead concentrations in 30 non-occupationally exposed men between the ages of 23 and 73 showed an annual increment of $0.46 \mu \mathrm{g} / \mathrm{g}$ bone mineral/year. The mean deviation from the regression of tibia lead upon age was $3.5 \mu \mathrm{g} / \mathrm{g}$ bone mineral. Tibia lead concentration in one subject with a history of exposure to lead was 69.6 (SD 3.5 ) $\mu \mathrm{g} / \mathrm{g}$ bone mineral. The improved precision of the point source large detector system means that greater confidence can be placed on the results of in vivo measurements of lead concentration. This will allow studies of the natural history of non-occupational lead accumulation in normal subjects and should permit investigations of the efficacy of therapeutic interventions in subjects poisoned with lead.
\end{abstract}

(British fournal of Industrial Medicine 1993;50:637-641)

Lead has been recognised as an occupational hazard for a long time and was, in 1981, the first designated substance to be regulated in Ontario, Canada under the Occupational Health and Safety

Department of Nuclear Medicine, ChedokeMcMaster Hospitals

C L Gordon, C E Webber

Department of Physics and Astronomy, McMaster

University, Hamilton, Ontario, Canada

D R Chettle
Act. ${ }^{1}$ Recent exposure to metallic or inorganic lead can be assessed from measurements of the concentration of lead in blood. The retention time of absorbed lead in blood is short, however, and blood lead concentrations do not reflect long-term cumulative exposure. Because absorbed lead accumulates in the skeleton, a measure of lead concentration in bone is an indicator of cumulative exposure. ${ }^{23}$ Such exposures may be due to either or both environmental and occupational sources of lead.

In the past decade a number of $x$ ray fluorescence systems have been designed and constructed for the in vivo measurement of lead concentrations in bone. The first measurements used $122 \mathrm{keV} \gamma$ rays from a ${ }^{57} \mathrm{Co}$ source to excite characteristic lead $x$ rays from finger bones. ${ }^{4}$ This technique was later adopted and applied to population studies by others. ${ }^{5}$ Substitution of ${ }^{109} \mathrm{Cd}$ for ${ }^{57} \mathrm{Co}$ allowed measurements to be made at other bone sites such as the tibia ${ }^{6}$ and also allowed quantification in units of mass of lead per unit mass of bone mineral interrogated by the incident photon beam.

A recent comparison was made between the ${ }^{109} \mathrm{Cd}$ and ${ }^{57} \mathrm{Co}$ based systems in an industrially exposed Swedish population. This collaborative study showed that finger lead and tibia lead were strongly correlated and that higher measurement precision was achieved for tibia lead. ${ }^{7}$

In this paper we describe an upgraded ${ }^{109} \mathrm{Cd}$ system that uses a point source centred on the face of and shielded from a high purity germanium (HPGe) detector. The design differences between our system and the improved annular source version ${ }^{8}$ will be presented together with preliminary data gathered from a normal human population. Finally, to show the sensitivity of this new system, we report the result of a measurement on an occupationally exposed subject.

\section{Materials and methods}

The instrument for measuring lead in bone is based on a low energy HPGe detector and modular electronics supplied by Canberra-Packard Canada (Mississauga, Ontario, Canada, L4Z 2E8). The detector (model GL 2020R) has a sensitive volume (20 mm thick and $20 \mathrm{~cm}^{2}$ in cross sectional area) behind an entrance window of $0.5 \mathrm{~mm}$ beryllium. 
The detector cryostat geometry is of the horizontal integral type and the measured energy resolution for the $81 \mathrm{keV}$ photon from a low activity ${ }^{133} \mathrm{Ba}$ source is $550 \mathrm{eV}$. The detector output is fed through a model $2001 \mathrm{CP}$ cold FET preamplifier to a fast spectroscopy amplifier (model 2024). The analogue output of the amplifier is converted by a fast ADC (model 8077) and analysed in a PC based Canberra System 100 multichannel analyser (Everex model 3000L) operating within Microsoft Windows 3.0.

A $1.11 \mathrm{GBq}{ }^{109} \mathrm{Cd}$ source in an X1017/1 capsule was purchased from Amersham Canada (Oakville, Ontario, Canada, L6L 5T7). This capsule is constructed of stainless steel and has an external diameter of $3.2 \mathrm{~mm}$, a height of $3.25 \mathrm{~mm}$, and an exit window of $0.2 \mathrm{~mm}$ thickness. Figure 1 shows diagrammatically, a cylindrical capsule shield and a collimator, each machined from a tungsten alloy (type HD 18.5, MiTech Metals, Indianapolis, Indiana, USA, 46202). The capsule shield and collimator are threaded so that any one of three collimators with different bore lengths $(1 \cdot 5,5 \cdot 5$, and $12.0 \mathrm{~mm}$ ) could be used for a measurement. Each collimator is fitted with a $0.5 \mathrm{~mm}$ thick copper filter to absorb the $\mathrm{Ag} \mathrm{K} x$ rays associated with the decay of ${ }^{109} \mathrm{Cd}$. Normally the shortest collimator, as illustrated in figure 1 , is employed.

The source capsule within its shield was positioned at the centre and in front of the beryllium window of the HPGe detector by means of the detector mount shown diagrammatically in fig 2 . A cylindrical iron sleeve with a closed end of styrene slides over the horizontally mounted detector and is held in place by grub screws. The purpose of this sleeve is to protect the beryllium window. An outer iron sleeve slides over the inner cylinder and is
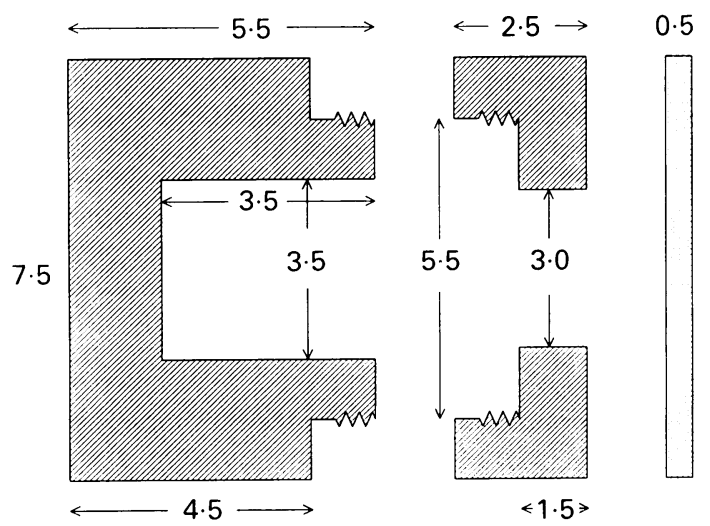

Figure 1 The tungsten shield, the short tungsten collimator and the copper filter for the ${ }^{109} \mathrm{Cd}$ source. All dimensions are $\mathrm{mm}$. again held in position with grub screws. The end of the outer cylinder contains slots into which four styrene spokes can fit. Both ends of each spoke are threaded. One end is screwed into an open ended lucite cylinder that holds the capsule shield as a friction fit. Threaded on to the other end of each spoke is a nut that allows for the adjustment of the position of the capsule shield in the plane parallel to the detector window. Adjustment of the depth of the spokes within each slot allows for sufficient movement of the source in a direction perpendicular to the detector window so that different collimators can be employed if required. A source shutter made from the tungsten alloy is mounted on an arm at the edge of the outer iron cylinder.

The HPGe detector, together with the ${ }^{109} \mathrm{Cd}$ source, is mounted on a table that allows for adjustment of the detector in three orthogonal directions. The subject to be measured sits comfortably in a chair and the table is adjusted to bring the copper face of the capsule shield within two to three $\mathrm{cm}$ of the skin over the anteromedial aspect of the left tibia. The leg is held in position against adjustable lucite plates by means of two cloth straps fastened around the leg, one above and one below the measurement site. If a measurement is made at the calcaneus, the source shield is positioned two to three $\mathrm{cm}$ from the lateral aspect of the right calcaneus.

To calibrate the system bone phantoms containing known concentrations of lead were constructed from plaster of Paris. The phantoms were cylindrical with an outer diameter of $4 \mathrm{~cm}$ and a central medullary canal of diameter $1.6 \mathrm{~cm}$ occupied by a bone marrow simulating rod of polyethylene.

For either a phantom bone or for each subject the spectrum was accumulated for $2000 \mathrm{~s}$ or until the counting error associated with the coherent photopeak was $1 \%$ or less. The data for each subject or calibration phantom was fitted by a gradient search least squares procedure. The optimum fit is achieved if a spectrum is divided into three regions, which are examined separately. In all three regions the peaks are fitted to gaussians of fixed width. Region 1 contains the $\mathrm{K} a_{1}$ and $\mathrm{K} a_{2} x$ rays with a Compton component that decreases rapidly with increasing energy. This background is best approximated by a double exponential. The background in region 2, which contains the $\mathrm{K} \beta_{1}$ and $\mathrm{K} \beta_{3} x$ rays, is approximated by a single exponential; the photoelectron bremsstrahlung edge features from calcium and phosphorus or sulphur are also modelled. ${ }^{9}$ The background in region 3 containing the coherent and $\mathrm{K} \beta_{2}$ peak is also fitted to a single exponential. $A$ common photopeak width in all three regions is used, which allows for a direct comparison of peak amplitudes when deriving lead concentrations.

Thirty male volunteers with no known occupational exposure to lead and with no apparent dis- 


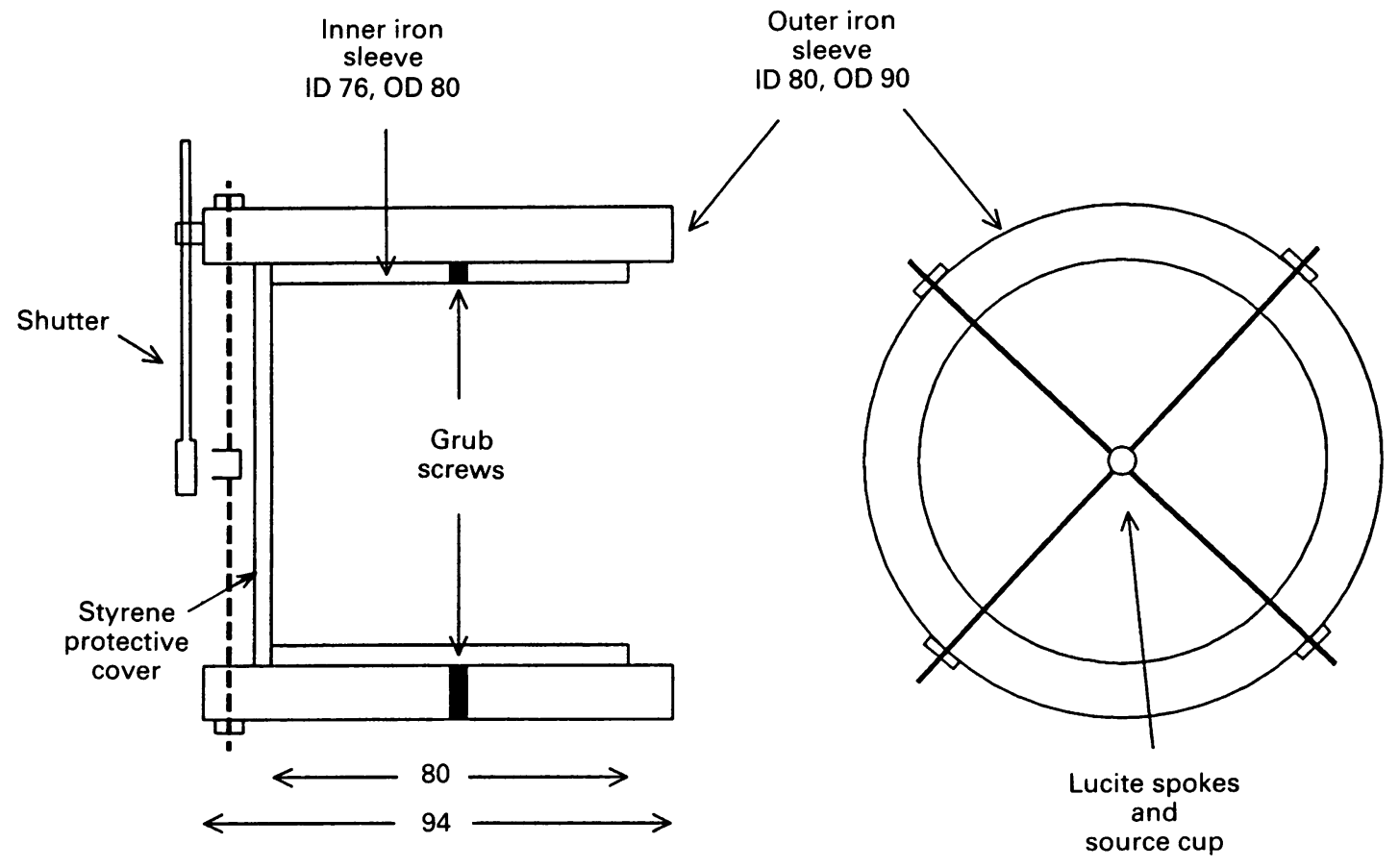

Figure 2 Detector mount for the ${ }^{109} \mathrm{Cd}$ source holder. All dimensions are $\mathrm{mm}$.

ease likely to affect calcium metabolism were recruited from hospital and university staff. Tibia measurements were performed as described. One male subject was recruited who had a known history of exposure to lead. Informed written consent was obtained before each measurement and the procedure was approved by the research ethics and project review board of McMaster University.

\section{Results}

Figure 3 shows a typical calibration curve based on the $\mathrm{K} a_{1} x$ ray peak. Each phantom was measured five times with repositioning between each measurement. The error bars shown in fig 3 represent 2 standard deviations (SDs) above plus 2 SDs below the mean. The correlation coefficient is 0.999 . The intercept is significantly different from 0 and suggests that the plaster of Paris contained some lead. Atomic absorption measurements gave a blank concentration of about $3 \mu \mathrm{g} / \mathrm{g}$.

Four other calibration curves could be derived based on the ratios of other $x$ ray photopeaks. The signal to noise ratio associated with each peak produces different precision errors after fitting. Concentrations derived from the $\mathrm{K} a_{1} x$ rays have the lowest associated error whereas those derived from the $\mathrm{K} \beta_{2} x$ rays have the highest. To maximise the spectral information contributing to the final determination of the lead concentration, an inverse variance weighted mean based on all peaks except $\mathrm{K} \beta_{2}$ is calculated. This weighted mean error, compared with that calculated from $\mathrm{Ka} a_{1}$ alone, is typically $3 \mu \mathrm{g} / \mathrm{g}$ bone mineral instead of $4.5 \mu \mathrm{g} / \mathrm{g}$. If the minimum detectable concentration is defined as twice this uncertainty, then the detection limit for our system is currently $6 \mu \mathrm{g} / \mathrm{g}$ bone mineral.

Figure 4 shows the results of in vivo measurements of tibia lead concentrations. The linear regression equation derived from non-occupationally exposed subjects was:

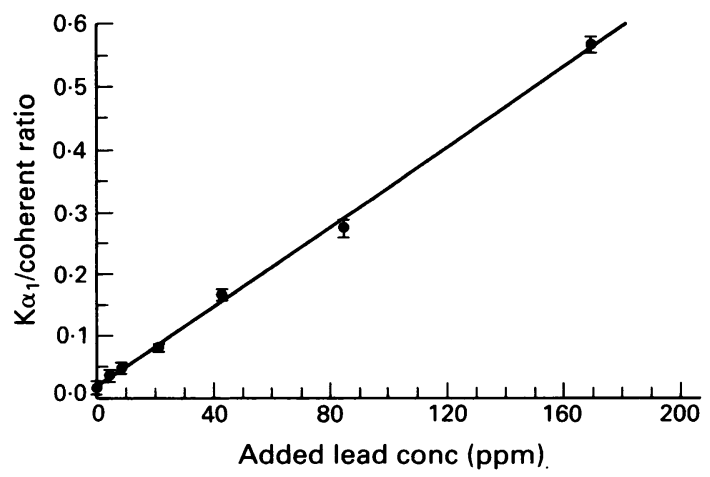

Figure 3 A typical calibration curve obtained from bone phantoms showing the ratio of $\mathrm{Ka}_{1}$ lead $x$ rays to coherently scattered $\gamma$-rays as a function of lead concentration. 


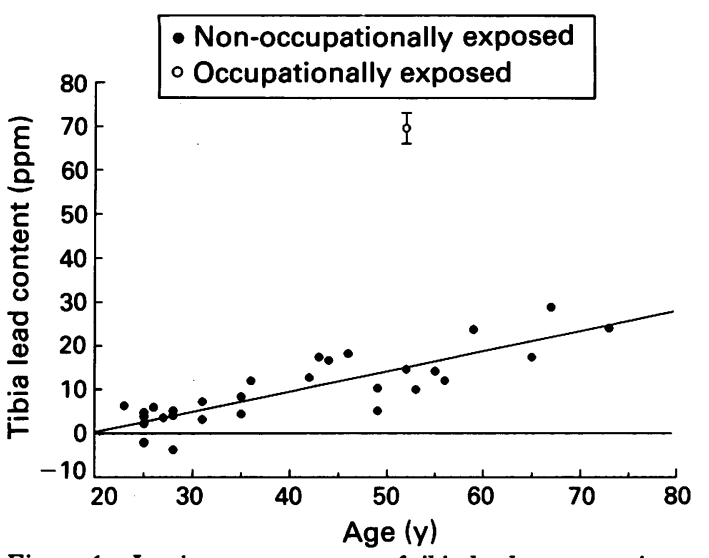

Figure 4 In vivo measurements of tibia lead concentration.

tibia lead $(\mu \mathrm{g} / \mathrm{g}$ bone mineral $)=$

$-8.97+0.463 \times$ age

with a correlation coefficient of 0.85 , an average weighted mean error of each measurement of $2.9 \mu \mathrm{g} / \mathrm{g}$, and a root mean square deviation of the points from the regression line of $3.5 \mu \mathrm{g} / \mathrm{g}$.

From the above regression equation, the expected value for the 52 year old, occupationally exposed subject was $15.1 \mu \mathrm{g} / \mathrm{g}$ bone mineral. The observed value was 69.6 (SD 3.5) $\mu \mathrm{g} / \mathrm{g}$ bone mineral.

One subject measured on our instrument had had previous measurements with an annular ${ }^{109} \mathrm{Cd}$ system. The result with the point source system was 12.7 (SD 2.9) $\mu \mathrm{g} / \mathrm{g}$ bone mineral. When measured nine years previously, with the earliest version of the annular system, the result was 11.7 (SD 8.8) $\mu \mathrm{g} / \mathrm{g}$ bone mineral.

\section{Discussion}

The major difference between this and earlier versions of instruments for measurement of lead in bone is that the previous arrangement of an annular source surrounding a small detector $(16 \mathrm{~mm}$ diameter, $7 \mathrm{~mm}$ thick) has been replaced by a large detector $(51 \mathrm{~mm}$ diameter, $20 \mathrm{~mm}$ thick) surrounding a point source. This leads to a considerable improvement in utilisation of source photons and allows reduction of the source activity from about $4 \mathrm{Gbq}$ to about $1 \mathrm{Gbq}$. With roughly the same source to sample separation and with comparable counting times, the detection limit of the point source system is improved by almost a factor of two compared with the annular system. ${ }^{8}$

The regression equation fitted to the control data suggests that for apparently unexposed men, tibia lead concentration increases by about $0.46 \mu \mathrm{g} / \mathrm{g}$ bone mineral/year. The rate of increase in our controls can be compared to previous results obtained both in vivo and in vitro. Tibia lead concentrations for 22 control subjects measured in the United Kingdom with an annular ${ }^{109} \mathrm{Cd}$ system gave a rate of increase of $0.60 \mu \mathrm{g} / \mathrm{g}$ bone mineral/year. ${ }^{6}$ Measurements on tibia samples obtained from 134 necropsies performed in Minnesota gave an age coefficient of $0.37 \mu \mathrm{g} / \mathrm{g}$ bone ash/year. ${ }^{10}$ For a group of 26 men and eight women from Massachusetts, the rate was $0.31 \mu \mathrm{g} / \mathrm{g}$ bone mineral/year. ${ }^{11}$

The mean measurement precision for the control subjects was $2.9 \mu \mathrm{g} / \mathrm{g}$ bone mineral whereas the population deviation from regression was $3.5 \mu \mathrm{g} / \mathrm{g}$ bone mineral. This implies that just about two thirds of the population variation in lead concentration in bone is due to measurement uncertainty with biological variation between subjects accounting for the remainder. The uncertainty in the measurement of the occupationally exposed subject was $3.5 \mu \mathrm{g} / \mathrm{g}$ bone mineral. The difference between the value found and a value predicted from the control data was about 16 times this uncertainty. At the same time, the maximum deviation of any of the controls from the fitted regression line was only about twice this uncertainty. A consequence of improved precision is that greater confidence can be placed on results such as those obtained with the point source system for the subject who has been measured previously with the annular system.

This work has shown that the use of a point source and a large area detector improves the precision of $\mathrm{K} x$ ray fluorescence excitation measurements of lead concentration in bone. The minimum detectable limit for the instrument we describe in this paper has been improved by almost a factor of two. The better precision of this system has increased the confidence with which raised concentrations of tibia lead can be identified. Further increases in precision may be possible if the volume of bone examined is increased by using multielement $\mathrm{HPGe}$ detectors with an array of point sources.

This work would not have been possible without an Occupational Health and Safety Award (253/R) from the Ontario Ministry of Labour. We are grateful for the machining skills of Michael Roberts.

Requests for reprints to: C E Webber, Department of Nuclear Medicine, Chedoke-McMaster Hospitals, 1200 Main Street West, Hamilton, Ontario, Canada, L8N 3Z5.

1 Ontario Ministry of Labour. Designated substances in the workplace: a guide to lead regulation. Ontario: Occupational Health and Safety Division, Ontario Ministry of Labour, July 1985 .

2 Christoffersson JO, Schutz A, Ahlgren L, Haeger- Aronsen B, Mattsson S, Skerfving S. Lead in finger bone analysed in vivo in active and retired lead works. $A m \mathcal{f}$ Ind Med 1984;6:447-57.

3 Somervaille LJ, Chettle DR, Scott MC, Tennant DR, 
McKiernan MJ, Skilbeck A, Trethowan WN. In vivo tibia lead measurements as an index of cumulative exposure in occupationally exposed subjects. $\mathrm{Br} \mathcal{F}$ Ind $\mathrm{Med}$ 1988;45: 174-81.

4 Ahlgren L, Mattsson S. An x-ray fluorescence technique for in vivo determination of lead concentration in a bone matrix. Phys Med Biol 1979;24:136-45.

5 Price J, Baddeley H, Kenardy JA, Thomas BJ, Thomas BW. In vivo $\mathrm{x}$-ray fluorescence estimation of bone lead concentrations in Queensland adults. Brit 7 Radiol 1984;57:29-33.

6 Somervaille LJ, Chettle DR, Scott MC. In vivo measurement of lead in bone using x-ray fluoresence. Phys Med Biol 1985;30:929-43.

7 Somervaille LJ, Nilsson U, Chettle DR, et al. In vivo measurements of bone lead-a comparison of two x-ray fluorescence techniques used at three different bone sites. Phys Med Biol 1989;34:1833-45.

8 Chettle DR, Scott MC, Somervaille LJ. Improvements in the precision of in vivo bone lead measurements. Phys Med Biol 1989;34:1295-1300.

9 Chettle DR. Photoelectron Bremsstrahlung-Analytical possibilities. Phys Med Biol 1990;35:259-64.

10 Wittmers LE, Aufderheide AC, Wallgren J, Alich A, Rapp G. Lead in bone: IV Distribution of lead in the human skeleton. Arch Environ Health 1988;43:381-91.

$11 \mathrm{Hu} \mathrm{H}$, Milder FL, Burger DE. X-ray fluorescence measurements of lead burden in subjects with low-level community léad exposure. Arch Environ Health 1990;45:335-41.

Accepted 7 September 1992 